\title{
Dissolution of calcium pyrophosphate crystals by polyphosphates: an in vitro and ex vivo study
} R Cini, D Chindamo, M Catenaccio, S Lorenzini, E Selvi, F Nerucci, M P Picchi, G Berti,
R Marcolongo

\begin{abstract}
Objective-To determine the dissolving ability (DA) of linear pentasodium tripolyphosphate (PSTP), cyclic trisodium metaphosphate (TSMP), polymeric sodium metaphosphate (SMP) on synthetic crystals of calcium pyrophosphate dihydrate (CPPD) and on crystalline aggregates of menisci from patients with chondrocalcinosis (CC).

Methods-Synthetic CPPD crystals were mixed with phosphate buffered saline (PBS), which contained the different polyphosphates, for one hour at $37^{\circ} \mathrm{C}$. The calcified menisci were obtained from the knees of four female patients with CPPD disease who underwent total arthroscopic meniscectomy for degenerative meniscal lesions. Meniscal cryosections and fragments were incubated in SMP $(15 \mathrm{mg} / \mathrm{ml}$ PBS) at $37^{\circ} \mathrm{C}$ for one hour and 24 hours, respectively. Histological evaluation on meniscal samples after polyphosphate incubation was carried out by ordinary transmitted light microscopy and polarised light microscopy. The dissolution of CPPD crystals by polyphosphates was assessed by atomic absorption spectroscopy, which determined the amount of calcium liberated from synthetic crystals and meniscal fragments. Cytotoxicity of SMP was evaluated by tetrazolium salt assay and by an ultrastructural study on cultured chondrocytes.
\end{abstract}

Results-SMP and PSTP showed higher DA on CPPD crystals than TSMP. Analysis of the DA values at increasing concentrations of SMP showed that a concentration of $15 \mathrm{mg} / \mathrm{ml}$ completely dissolved $2.0 \mathrm{mg}$ CPPD crystals. The solution of meniscal CPPD crystals showed a significant increase of calcium concentration after three hours and 24 hours of SMP incubation $(p=0.0001 ; \quad$ Kruskal-Wallis analysis of variance) compared with fragments incubated in PBS control solution. Macroscopic and microscopic evaluation of meniscal specimens showed a notable reduction of CPPD deposits. A $50 \%$ inhibitory dose on cultured chondrocytes was reached at the maximum concentration of SMP used in this work $(15 \mathrm{mg} / \mathrm{ml})$; ultrastructural analysis did not show morphological alterations in the treated cells. Conclusion-The results of this study indicate that linear polyphosphates are effective in dissolving both synthetic and ex vivo CPPD crystal aggregates. This suggests a potential therapeutic use for these molecules in the treatment of symptomatic CC.

(Ann Rheum Dis 2001;60:962-967)

Chondrocalcinosis (CC) is a disease characterised by crystalline deposits of calcium pyrophosphate dihydrate (CPPD; $\left.\left[\mathrm{Ca}_{2}\left\{\mathrm{O}\left(\mathrm{PO}_{3}\right)_{2}\right\}\right]\left(2 \mathrm{H}_{2} \mathrm{O}\right)\right)$, which tend to form solid aggregates within the articular cartilage, the synovial membrane, the articular capsule, the tendons, and the ligaments. Crystal shedding in the synovial cavity is thought to trigger acute arthritis (pseudogout). CPPD crystals were first discovered by McCarty some 40 years ago in the synovial fluid of a patient who had recurrent arthritis. ${ }^{1}$ Other reports confirmed the role of CPPD crystalline aggregates in setting off an inflammatory response. ${ }^{2}{ }^{3} \mathrm{~A}$ number of studies have been performed to evaluate the dissolution of CPPD crystals both in vitro and in vivo, but an effective and safe treatment is yet lacking. ${ }^{4-8}$ Bennett and colleagues observed that joint lavages with calcium chelating agents including sodium EDTA could dissolve CPPD crystals in patients with $\mathrm{CC}$ and recurrent bouts of pseudogout; nevertheless, all the experiments provoked a pseudogout attack. ${ }^{6}$ Oral magnesium has been reported to lessen the inflammatory manifestations of CPPD crystal related arthropathy, and there was no evidence of decreased deposits. ${ }^{78}$

Our study aimed at estimating the dissolving ability (DA) values of some phosphatecontaining molecules, particularly linear and cyclic phosphates, on CPPD crystals in aqueous systems under physiological conditions (pH 7.4 and osmolality in the range 280-300 $\mathrm{mmol} / \mathrm{kg}$ ). These compounds were chosen because their chelating ability towards metal ions has been known for decades and because previous research showed that chain polyphosphates (such as the Graham salts) reduced aortic calcifications in rats. ${ }^{9}$

Materials and methods

MATERIALS

Cyclic $\mathrm{Na}_{3}\left(\mathrm{PO}_{3}\right)_{3}$, linear $\mathrm{Na}_{5}\left\{\mathrm{O}\left(\mathrm{PO}_{3}\right)_{3}\right\} \cdot 6 \mathrm{H}_{2} \mathrm{O}$, $\mathrm{CaCl}_{2}, \mathrm{MgCl}_{2}, \mathrm{NaCl}$, Dulbecco's calciummagnesium free phosphate buffered saline (PBS), Dulbecco's modified Eagle's medium (DMEM), clostridium collagenase, tetrazolium salt 3-(4,5-dimethylthiazol-2-yl)-2,5diphenyltetrazolium bromide (MTT), dimethyl sulphoxide were all Sigma (Italy) analytical grade products. Linear $\mathrm{Na}_{\mathrm{n}}\left(\mathrm{PO}_{3}\right)_{\mathrm{n}}$ was from $\mathrm{C}$ 
A

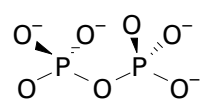

C

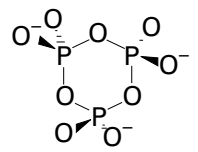

B

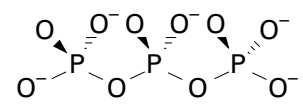

D

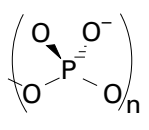

Figure 1 Structural formulas for $(A)$ pyrophosphate, $\mathrm{O}\left(\mathrm{PO}_{3}\right)_{2}^{4-} ;(\mathrm{B})$ a linear tripolyphosphate, $\mathrm{O}\left(\mathrm{PO}_{3}\right)_{3}^{5-} ;(\mathrm{C})$ cyclic trimetaphosphate, $\left(\mathrm{PO}_{3}\right)_{3}^{3-} ;(\mathrm{D})$ polymeric metaphosphate $\left(\mathrm{PO}_{3}^{-}\right)_{n^{*}}$.

Erba (Italy). All compounds were used as purchased without any further purification. Figure 1 shows selected structural formulas.

IN VITRO TESTS

CPPD synthesis

Triclinic and monoclinic CPPD crystals were prepared as described elsewhere. ${ }^{10}$ As the dissolving rate of crystals in fluids is related, among other things, to the size of the solid particles, all synthetic CPPD crystals were prepared rigorously under the same conditions to ensure size uniformity. The triclinic and monoclinic crystal phase and the quality of each CPPD crystal preparation were determined by powder $x$ ray diffraction analysis; the average crystal sizes were checked with a polarised light microscope. The crystals (needlerhomboid shaped) had average dimensions of 1-30 $\mu \mathrm{m}$ for all preparations.

\section{Determination of the $D A$ values for CPPD} crystals

The solutions of the polyphosphates were prepared by mixing a weighed amount of the compounds with PBS. The $\mathrm{pH}$ and osmolality were checked before the addition of the CPPD crystals for all the solutions.

Samples of the air dried CPPD crystals (2.0 mg each) were mixed with PBS $(5.0 \mathrm{ml}$ each), which contained the polyphosphate. The mixtures were allowed to remain at $37^{\circ} \mathrm{C}$ for one hour with continual stirring. The mixtures were then filtered through syringes equipped with membrane surfactant-free cellulose acetate Nalge Nunc International NALGENE filters (disk diameter $25 \mathrm{~mm}$; mean hole diameter 0.22 $\mu \mathrm{m})$. The filtrates, stored in polythene test tubes, were then analysed for the calcium concentration, which was measured by a Perkin-Elmer Analyst 100 atomic absorption spectrophotometer. All experiments were performed three times and the mean (SD) values were expressed as $\mu \mathrm{g} \mathrm{Ca}^{2+} / \mathrm{ml}$ after correction for the calcium contents in the dissolving solutions which were not mixed with CPPD crystals.

EX VIVO TESTS

To evaluate the DA of the polyphosphate on the tissue aggregates of CPPD we carried out solubilisation tests on the calcified menisci. The menisci were obtained from four female patients (mean (SD) age 60 (3) years) with

Table 1 Analytical data for the dissolution of $2 \mathrm{mg}$ calcium pyrophosphate dihydrate crystals in different polyphosphate samples

\begin{tabular}{lll}
\hline & $\begin{array}{l}\text { DA valuest }(S D) \\
\text { Dissolving solution * }\end{array}$ & Dissolution (\%) \\
\hline $1.5 \mathrm{mg} \mathrm{SMP} \ddagger / \mathrm{ml}$ & $24.1(3)$ & 21.9 \\
$1.5 \mathrm{mg} \mathrm{TSMP} \ddagger / \mathrm{ml}$ & $1.6(0.5)$ & 1.5 \\
$2.3 \mathrm{mg} \mathrm{PSTP} \ddagger / \mathrm{ml}$ & $21.7(4.3)$ & 19.7
\end{tabular}

*The amount of the polyphosphates corresponds to a concentration of $14.7 \mathrm{mM}$ phosphate in phosphate buffered saline $(280-300 \mathrm{mmol} / \mathrm{kg}$, pH 7.4).

†Dissolving ability (DA) values were expressed as concentrations of calcium ions present in solution after one hour of incubation at $37^{\circ} \mathrm{C}$.

$\ddagger \mathrm{SMP}=$ sodium metaphosphate TSMP = trisodium metaphosphate; PSTP $=$ pentasodium tripolyphosphate.

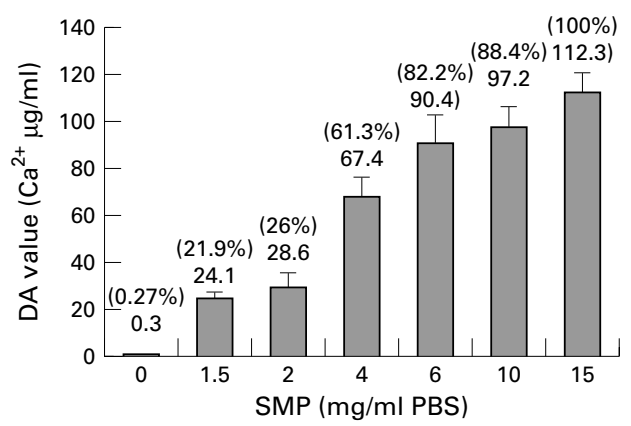

Figure 2 Effect of increasing concentrations of polymeric sodium metaphosphate (SMP) on the solubilisation of synthetic CPPD crystals ( $2 \mathrm{mg}$ ) after one hour of incubation at $37^{\circ} \mathrm{C} . \mathrm{DA}=$ dissolving ability. The bars show the mean (SD) of DA values obtained by three separate experiments. Values in brackets show the percentage of dissolution.

degenerative meniscal lesions who had undergone a total arthroscopic meniscectomy. All these patients had CC as diagnosed by knee $x$ ray and synovial fluid analysis. In particular, the $x$ rays showed linear opacities in the joint spaces, whereas synovial fluid analysis disclosed birefringent positive CPPD crystals.

Immediately after arthroscopy, the menisci were rinsed twice in physiological solution $(0.9 \% \mathrm{NaCl})$. Every sample was photographed as a whole and then cut into three parts of equal dimensions $(\sim 2 \times 1.5 \times 0.5 \mathrm{~cm})$. One of these fragments was snap frozen in isopentane, kept at $-80^{\circ} \mathrm{C}$, and used for the microscopic evaluation of the dissolution of CPPD deposits.

\section{Microscopic study}

Transverse $7 \mu \mathrm{m}$ sections were observed under ordinary transmitted and polarised light microscope and photographed before and after incubation in polymeric sodium metaphosphate (SMP) $(15 \mathrm{mg} / \mathrm{ml} \mathrm{PBS})$ for one hour at $37^{\circ} \mathrm{C}$. The negative controls were obtained by incubating a section of each sample in PBS.

\section{Macroscopic study}

This study was carried out on the other two fragments. These were photographed, incubated in SMP (15 mg $/ \mathrm{ml} \mathrm{PBS}$ ) and photographed again after one hour, three hours, and 24 hours of treatment. At the same time intervals, a $1 \mathrm{ml}$ aliquot from each solution was filtered and subsequently tested for calcium concentration by atomic absorption spectroscopy. Statistical analysis of the data was carried out by Kruskal-Wallis analysis of variance on ranks. Significance was established at $\mathrm{p}<0.05$. 


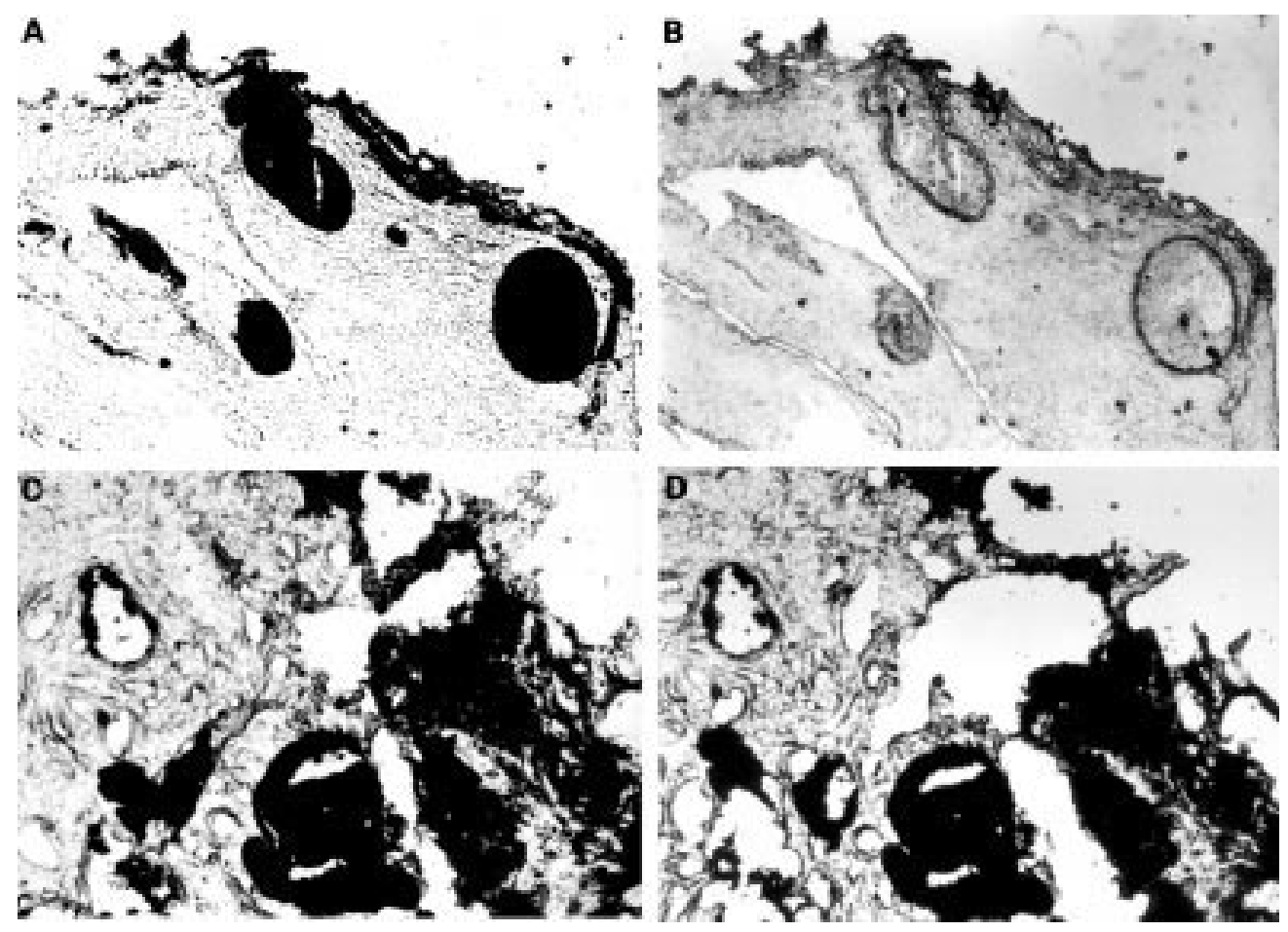

Figure 3 A calcified human meniscal section $(7 \mu \mathrm{m})(A)$ before treatment; (B) after one hour of treatment with 15 mg sodium metaphosphate (SMP)/ml phosphate buffered saline (PBS), the crystalline aggregates of calcium pyrophosphate dihydrate (CPPD) are dissolved; (C) before treatment; (D) after one hour of treatment with PBS solutions, the crystalline aggregates of CPPD are not dissolved. (Ordinary transmitted light microscopy original magnification $\times 10$.)

At the end of the 24 hours' incubation, the fragments were cut at the mid-line and the resulting two fragments of each sample were snap frozen. Cryosections ( $7 \mu \mathrm{m}$ each) were collected on slides and observed under transmitted and polarised light microscopy. Finally, the incubating solutions were centrifuged at $700 \mathrm{~g}$ for 10 minutes, and the resulting pellets were observed under polarising microscopy to evaluate the presence of CPPD crystals liberated from meniscal fragments. Negative controls were obtained by incubating fragments of each sample in PBS.

\section{Chondrocyte cytotoxicity determinations}

Human articular cartilage was obtained from the femoral heads of four osteoarthritic subjects (mean (SD) age 67.5 (2.5) years) undergoing surgery for total hip prostheses. Immediately after surgery, macroscopically healthy cartilage was removed in aseptical conditions and minced into $2 \mathrm{~mm}^{3}$ pieces. The cartilage fragments were washed in saline solution (140 $\mathrm{mM} \mathrm{NaCl}, 5 \mathrm{mM} \mathrm{KCl}, 5 \mathrm{mM}$ glucose, $10 \mathrm{mM}$ HEPES, pH 7.4) containing $200 \mathrm{U} / \mathrm{ml}$ penicillin and $200 \mu \mathrm{g} / \mathrm{ml}$ streptomycin. The cartilage was then digested by clostridial collagenase 1 $\mathrm{mg} / \mathrm{ml}$ in PBS containing the same concentration of antibiotics. The collagenase digestion was carried out at $37^{\circ} \mathrm{C}$ for $14-18$ hours with moderate stirring. The solution was then filtered, washed in saline solution, and centrifuged for 10 minutes at $700 \mathrm{~g}$. As shown by the trypan blue viable stain, $90-95 \%$ of the cells recovered were alive. Chondrocyte cells were plated out in $200 \mu \mathrm{l}$ of DMEM supplemented with L-glutamine $(2 \mathrm{mM})$, fetal calf serum $(10 \%)$, penicillin $(200 \mathrm{U} / \mathrm{ml})$, and streptomycin
$(200 \mu \mathrm{g} / \mathrm{ml})$ in 96 well microtitre plates $\left(10^{4}\right.$ cells/well) and allowed to attach for 24 hours $\left(37^{\circ} \mathrm{C}, 5 \% \mathrm{CO}_{2}\right)$. Cells were then incubated with an increasing concentration of SMP in PBS ( $\mathrm{pH} 7.4 ; 280-300 \mathrm{mmol} / \mathrm{kg}$ ) for 24 hours (six wells for each SMP concentration). The control culture was obtained by incubating cells with PBS for 24 hours. Cytotoxicity was determined two days after exposure to SMP by the MTT assay, which measures the number of metabolically active cells by a colorimetric technique, as reported elsewhere. ${ }^{11}$ Results were expressed as mean (SD).

\section{Transmission electron microscopy}

The human chondrocytes, treated for 24 hours with increasing concentrations of SMP, were mechanically detached from the wells, rinsed in PBS, centrifuged at $700 \mathrm{~g}$ for 10 minutes, fixed for two hours at $4^{\circ} \mathrm{C}$ in cold Karnovsky fixative, ${ }^{12}$ rinsed overnight in $0.1 \mathrm{M} \mathrm{pH} 7.2$ cacodylate buffer, and post-fixed for one hour at $4^{\circ} \mathrm{C}$ in $1 \%$ buffered $\mathrm{OsO}_{4}$, dehydrated in a graded series of ethanol, and embedded in Epon-Araldite. Ultrathin sections cut with an LKB III ultramicrotome were collected in copper grids, stained with uranyl acetate and lead citrate, and then photographed with a Philips CM10 electron microscope. For the ultrastructural examination about 30 chondrocytes for each patient were observed by transmission electron microscopy.

COMPLEX FORMATION ENERGIES

All density functional calculations were performed using the Gaussian94/DFT package on an Origin 2000 SG machine. ${ }^{13}$ Geometry 

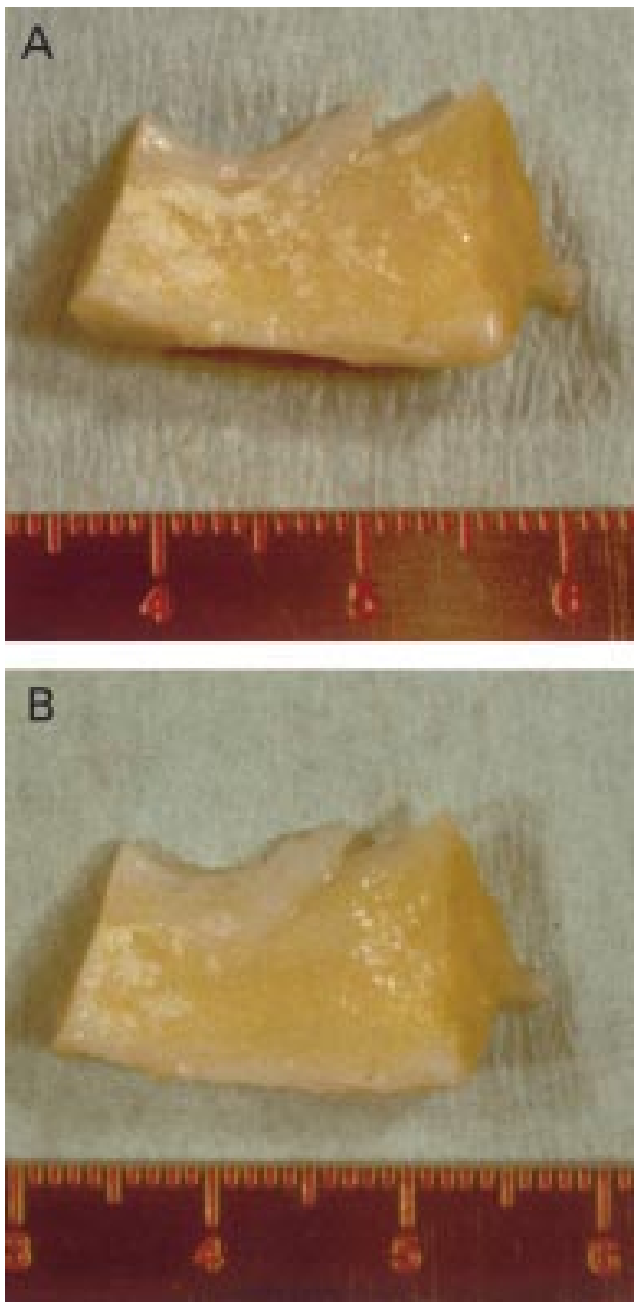

Figure 4 Calcified human meniscus fragments $(A)$ before treatment; (B) after 24 hours of incubation with $15 \mathrm{mg}$ sodium metaphosphate (SMP)/ml phosphate buffered saline (PBS), the crystalline aggregates of calcium pyrophosphate dihydrate (CPPD) are significantly decreased.

optimisations and energy calculations were performed using the B3LYP method, and the LANL2DZ basis set; the $6-31 \mathrm{G}^{\star \star}$ like functions were used for $\mathrm{P}$ atoms (for the methods, basis sets, and other details see Cini et $a l^{14-16}$ and the references cited therein).

\section{Results}

IN VITRO TESTS

Linear phosphates (SMP and linear pentasodium tripolyphosphate (PSTP)) showed higher DA values on CPPD crystals than cyclic phosphates (table 1). These data pertain to solutions of polyphosphates in PBS $14.7 \mathrm{mM}$ in $\mathrm{PO}_{3}$ unit: this allows a direct comparison

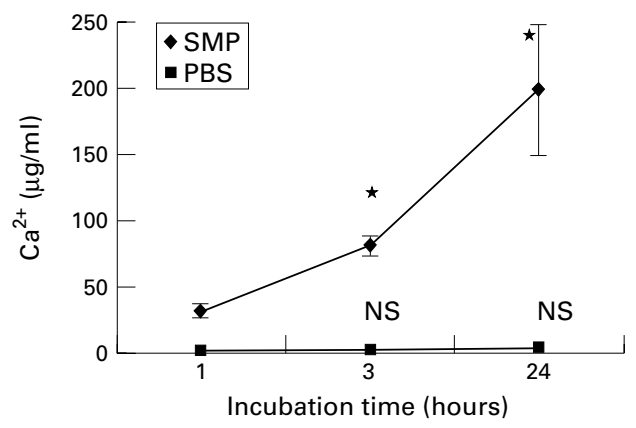

Figure 5 Variations of calcium concentrations after one hour, three hours, and 24 hours by incubating meniscal samples $(n=4)$ in sodium metaphosphate (SMP) (15 $\mathrm{mg} / \mathrm{ml})$ and phosphate buffered saline (PBS) $(p=0.0001$ Kruskal-Wallis analysis of variance). ${ }^{\star} p<0.05:$ SMP 24 hours v SMP three hours; SMP 24 hours v SMP one hour; SMP three hours $v$ SMP one hour; NS = not significant.

between different polyphosphates (the solutions have $\mathrm{pH} 7.4$ and osmolality in the 280-300 $\mathrm{mmol} / \mathrm{kg}$ range). The ionic strength of these solutions is due mostly to PBS, and is not significantly influenced by the equilibria which involve the ligands and the dissolution of CPPD crystals. Analysis of the DA values at increasing concentrations of SMP carried out after one hour of incubation showed that a concentration of $15 \mathrm{mg} / \mathrm{ml}$ PBS completely dissolved $2.0 \mathrm{mg}$ of CPPD crystals (fig 2).

\section{EX VIVO TESTS}

The CPPD crystalline aggregates in all meniscal sections from patients with CC disappeared within one hour from the onset of the incubation in SMP (15 mg/ml PBS), whereas no CPPD crystal dissolution was noted in the sections treated with PBS only (fig 3).

The macroscopic appearance of the meniscal fragments before and after 24 hours of treatment with SMP (15 mg/ml PBS) showed a net reduction of these crystalline aggregates (fig 4). Furthermore, mid-line sections showed numerous empty lacunae as compared with control PBS treated samples in the superficial and sublining meniscal layers. Transmitted and polarised light microscopy of the pellets from each incubating solution did not disclose birefringent positive CPPD crystals.

The calcium concentration liberated from the calcified meniscal fragments significantly increased after one hour, three hours, and 24 hours of SMP incubation ( $\mathrm{p}=0.0001$, KruskalWallis analysis of variance; fig 5).

\section{Chondrocyte cytotoxicity}

The results of preliminary cytotoxicity tests on human chondrocytes at increasing concentrations of SMP in PBS (pH 7.4, 280-300 mmol/

Table 2 Effect of increasing the concentration of polymeric sodium metaphosphate (SMP) on human chondrocytes by tetrazolium salt (MTT) assay

\begin{tabular}{llllll}
\hline \multicolumn{5}{c}{ SMP solution * $(m g / m l)$} & \\
\cline { 2 - 6 } & 0 & 1 & 2 & 6 & 15 \\
\hline Metabolically active cells (mean (SD)) + & 100 & $95(3.2)$ & $91(3.6)$ & $59.4(4.3)$ & $50(7.6)$ \\
\hline
\end{tabular}

*SMP was dissolved in phosphate buffered saline (PBS; 280-300 mmol/kg, $\mathrm{pH} 7.4$ ).

tValues were expressed as a percentage of metabolically active cells as compared with controls.

MTT test measures the number of metabolically active cells by a colorimetric technique. The data represent the mean results of four separate experiments. The control cultures were obtained by incubating cells with PBS for 24 hours. 

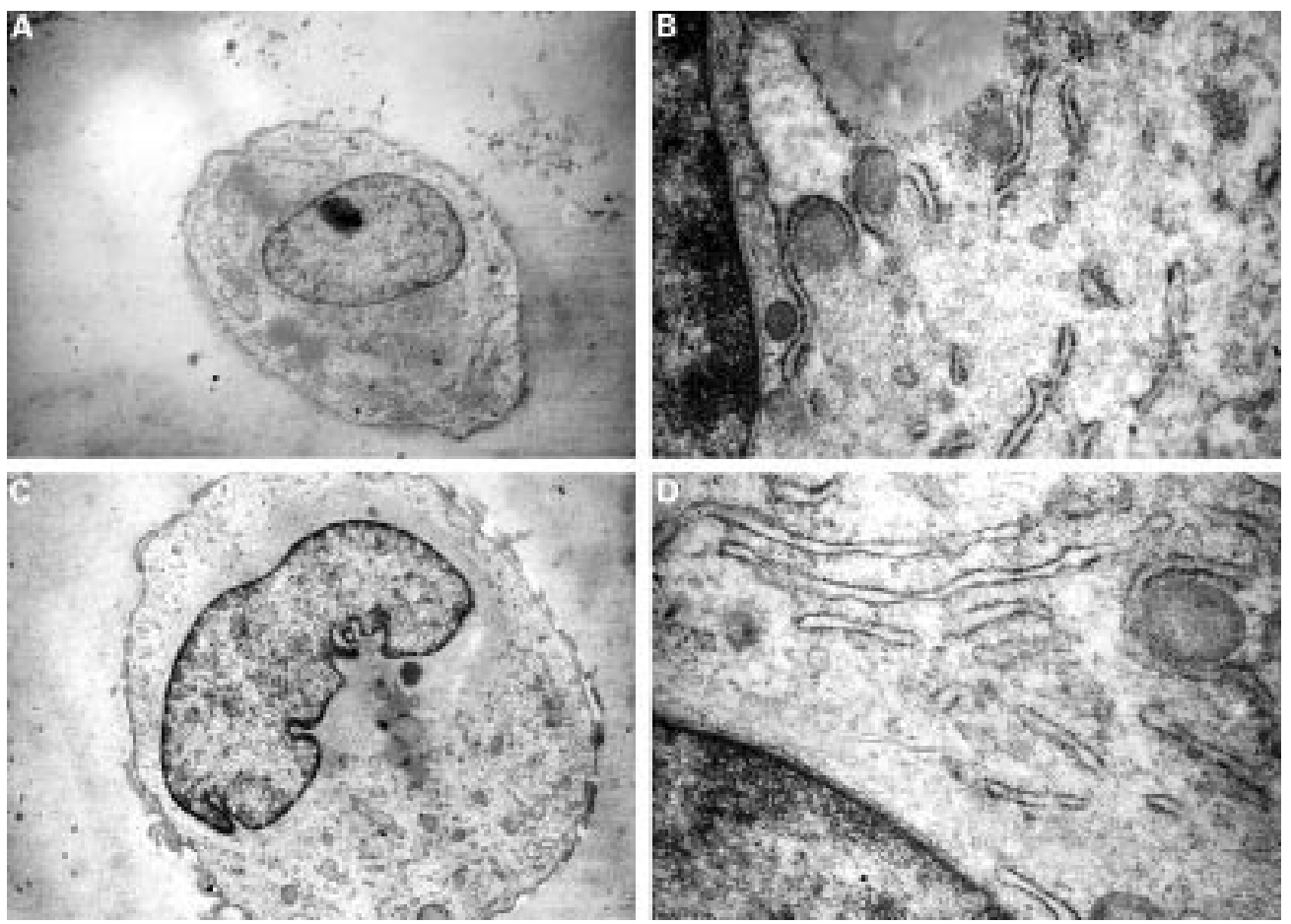

Figure 6 Transmission electron microscopy of human chondrocytes cultured in vitro: before $(A, C)$ and after $(B, D)$ treatment (24 hours of treatment with $15 \mathrm{mg}$ sodium metaphosphate (SMP)/ml phosphate buffered saline (PBS)); cells did not shown structural abnormalities, including the capsule, the pericellular matrix, the cellular membrane, the mitochondria, the inclusions, the smooth and rough reticulum, the Golgi, the lysosomes, and the nucleus. (Original magnification figs $A$, $C$ $\times 6000$; figs $B, D \times 20$ 000.)

$\mathrm{kg}$ ), showed that the $50 \%$ inhibitory dose was reached at the maximum concentration (15 $\mathrm{mg} / \mathrm{ml}$ ) used in this work (table 2).

Morphological evaluation by transmission electron microscopy showed no structural abnormalities at any site, including the capsule, the pericellular matrix, the cellular membrane, the mitochondria, the inclusions, the smooth and rough reticulum, the Golgi, the lysosomes, and the nucleus (fig 6).

\section{COMPLEX FORMATION ENERGIES}

The structures for pyrophosphate $\mathrm{O}\left(\mathrm{PO}_{3}\right)_{2}{ }^{4-}$, linear pentasodium tripolyphosphate (PSTP) $\mathrm{O}\left(\mathrm{PO}_{3}\right)_{3}{ }^{5-}$, cyclic trisodium metaphosphate (TSMP) $\left(\mathrm{PO}_{3}\right)_{3}{ }^{3-}$ anions, of the respective complexes with calcium $\left[\mathrm{Ca}\left\{\mathrm{O}\left(\mathrm{PO}_{3}\right)_{2}\right\}\right]^{2-}$, $\left[\mathrm{Ca}\left\{\mathrm{O}\left(\mathrm{PO}_{3}\right)_{3}\right\}\right]^{3-}$, and $\left.\left[\mathrm{Ca}\left(\mathrm{PO}_{3}\right)_{3}\right\}\right]^{-}$were all fully optimised. A detailed analysis of selected structures has been reported elsewhere. ${ }^{16}$ The agreement between observed and computed structures was satisfactory. Table 3 reports the energies for selected formal reactions. The absolute value of computed molar formation energy for calcium pyrophosphate $(-865.951$ $\mathrm{kcal}$, table 3; for the formal reaction at the gas phase) is much smaller than that for the formation of calcium PSTP (-982.342 kcal). This is related mostly to the larger overall charge of the $\mathrm{O}\left(\mathrm{PO}_{3}\right)_{3}{ }^{5-}$ compared with that of $\mathrm{O}\left(\mathrm{PO}_{3}\right)_{2}{ }^{4-}$.

Table 3 Complex formation energies ( $\mathrm{kcal} / \mathrm{mol}$ ) for selected formal reactions

\begin{tabular}{ll}
\hline Reaction & $-\Delta E$ (kcal) \\
\hline $\mathrm{Ca}^{2+}+\mathrm{O}\left(\mathrm{PO}_{3}\right)_{2}{ }^{4-} \rightarrow\left[\mathrm{Ca}\left\{\mathrm{O}\left(\mathrm{PO}_{3}\right)_{2}\right\}\right]^{2-}$ & 865.951 \\
$\mathrm{Ca}^{2+}+\left(\mathrm{PO}_{3}\right)_{3}{ }^{3-} \rightarrow\left[\mathrm{Ca}(\mathrm{PO})_{3}\right]^{-}$ & 637.331 \\
$\mathrm{Ca}^{2+}+\mathrm{O}\left(\mathrm{PO}_{3}\right)_{3}{ }^{5-} \rightarrow\left[\mathrm{Ca}\left\{\mathrm{O}\left(\mathrm{PO}_{3}\right)_{3}\right\}\right]^{3-}$ & 982.342 \\
\hline
\end{tabular}

The complex formation energy for calcium TSMP is $637.331 \mathrm{kcal}$. The computed formation energy does not take into account many important factors, such as the entropic contributions and the solvation effects; thus it was not possible to find a sound correlation between the formation energies and the DA values for the ligands. However, it is worth noting that calcium PSTP has a complex formation energy which is approximately $116 \mathrm{kcal}$ greater than that of pyrophosphate, and a high DA value on CPPD crystals.

\section{Discussion}

An examination of our in vitro tests (table 1) showed that the linear or branched chains built up by at least three $\mathrm{PO}_{3}$ units had large DA values for the CPPD crystals. In fact, DA values in the range $21.7-24.1 \mathrm{Ca}^{2+} \mu \mathrm{g} / \mathrm{ml}$ were measured for linear SMP and PSTP at $14.7 \mathrm{mM} \mathrm{PO}_{3}$ in PBS. Thus there is no significant influence on DA values when the phosphate chain is lengthened beyond three $\mathrm{PO}_{3}$ units. Interestingly, the DA value for the cyclic TSMP $\left(1.6(0.5) \mathrm{Ca}^{2+}\right.$ $\mu \mathrm{g} / \mathrm{ml})$, is much smaller than that of linear PSTP (21.7 (4.3) $\left.\mathrm{Ca}^{2+} \mu \mathrm{g} / \mathrm{ml}\right)$. In addition, the presence of the phosphates contained in PBS decreases the hydrolysis of the phosphatecontaining ligands.

The reason for the trend in the DA values lies, at least in part, in the complexing ability of the ligand molecules towards the calcium ion. The linear triphosphate chain seems to be particularly suited to linking a $\mathrm{Ca}^{2+}$ ion, whereas the very small DA values for cyclic TSMP suggest that the rigidity of the $\left(\mathrm{PO}_{3}\right)_{3}{ }^{3-}$ molecule, combined with its lower negative charge (when 
compared with $\mathrm{O}\left(\mathrm{PO}_{3}\right)_{3}{ }^{5-}$ and $\left.\mathrm{O}\left(\mathrm{PO}_{3}\right)_{2}{ }^{4-}\right)$, does not allow a linkage to $\mathrm{Ca}^{2+}$ that is strong enough to remove the metal cation from the surface of CPPD crystals. Calculations of the formation energies of complexed ligands to calcium ions have allowed us to investigate the different behaviour of the polyphosphates used in our study. Broadly speaking, lower formation energies correspond with a higher binding stability. More specifically, PSTP, which effectively dissolves CPPD crystals, corresponds with the higher binding stability to the calcium ion compared with that of TSMP and pyrophosphate. These findings highlight the greater dissolving ability of PSTP as compared with TSMP in removing the calcium ion from the pyrophosphate of the CPPD crystals.

The similarity found in the DA values of the linear PSTP and SMP ligands shows that the complex formation with a $\mathrm{Ca}^{2+}$ ion requires mostly only three consecutive $\mathrm{PO}_{3}$ units. Previous in vitro investigations have shown that triphosphate is the predominant metabolic product of soluble polyphosphate in yeast. ${ }^{17}$ This observation suggests that treatment carried out with SMP or other linear metaphosphate polymers/oligomers should maintain a high DA, even though a significant hydrolytic dissociative process takes place in vivo.

Of all these ligands, linear SMP has been shown to be the most efficacious dissolving agent because, compared with the others, it was possible to increase PBS concentrations while remaining in the range of physiological conditions. In particular, the PBS concentration of $15 \mathrm{mg} / \mathrm{ml}$ can be considered the highest that can be used, as it reaches the limit value of $\mathrm{pH}(7.4)$ and osmolality $(300 \mathrm{mmol} / \mathrm{kg})$. For this reason, we evaluated the DA of SMP on biological samples (human calcified menisci). Under these conditions, SMP displayed a significant DA, in that it worked not only on superficial calcifications but also more deeply, apparently without altering the normal histological structure of the meniscal fibrocartilage.

It has been noted that knee lavage with potent calcium chelating agents, such as disodium EDTA, may induce attacks of pseudogout. ${ }^{6}$ This has led to speculation that acute attacks may be the result of crystal shedding, promoted by the partial dissolution of superficial cartilaginous crystal aggregates. In view of this, we cannot exclude the possibility that the injection of phosphates into a joint might trigger an acute pseudogout attack. On the other hand, we found that phosphates in 24 hours of incubation can slowly remove most CPPD crystals from meniscal specimens without significant cellular toxicity.

Hence, our findings lend support to the idea that phosphates injected intra-articularly might induce a gradual dissolution of the crystal aggregates and of the CPPD crystals shed from the joint surface, thus preventing pseudogout attacks. Furthermore, the absence of CPPD crystals from the incubating solutions seems to confirm the ability of SMP to dissolve them completely once they are removed from the tissue matrix. These observations support our hypothesis that phosphates could be used to remove both free and tissue bound crystals without promoting acute pseudogout attacks. Hence, our data may have therapeutic implications, as at least some of the molecules studied could potentially be used for intra-articular treatment in CC, which so far can only be treated symptomatically. Further data are needed to investigate the feasibility of treatment of CC with these compounds.

Professor R Bargagli, Department of Environmental Biology, University of Siena, is gratefully acknowledged for most of the atomic absorption analyses. RC, thanks the University of Siena, Ministero dell'Universita e della Ricerca Scientifica e Tecnologica (MURST, Roma); Dr P Trada, Institute of Orthopedy, University of Siena, is acknowledged for supplying the menisci samples for the ex vivo experiments. Many thanks to Dr Nicolo, Pipitone for helpful comments.

Grant: CINECA (Centro Interuniversitario di Calcolo Elettronico, Bologna), 99/1522-5; Consiglio Nazionale delle Ricerche (CNR, Roma)

1 McCarty DJ Jr, Kohn NN, Faires JS. The significance of calcium phosphate crystal in the synovial fluid of arthritis patients: the pseudogout syndrome. Clinical aspects. Ann Intern Med 1962;56:711-37.

2 Denko CW, Withehouse WM. Experimental inflammation induced by naturally occurring microcrystalline calcium salts. J Rheumatol 1976;3:54-63.

3 Naccache PH, Bourgoin S, Plante E, Roberge CJ, De Medicis R, Lussier A, et al. Crystal induced neutrophil activation: evidence for the activation of phosphatidylcholine specific phospholipase D. Arthritis Rheum 1993;36: line specifi $117-25$.

$4 \mathrm{Xu}$ Y, Cruz T, Cheng PT, Pritzeker KPH. Effects of pyrophosphatase on dissolution of calcium pyrophosphate dihydrate (CPPD) crystals. J Rheumatol 1991;18:66-71

5 Von Linthoudt D, Solani I, Tender R, Locatelli P, Ott H, Schumacher HR. Citrate in synovial fluid and its relation to inflammation and crystal presence. J Rheumatol 1996;23: 3502-5.

6 Bennett RM, Lehr JR, Daniel J. Crystal shedding and acute pseudogout (an hypothesis based on therapeutic failure). Arthritis Rheum 1976;19:93-7.

7 Doherty M. Pyrophosphate arthropathy-recent clinical advances. Ann Rheum Dis 1983;42:38-44.

8 Patel KJ, Weidensaul D, Palma C, Ryan LM, Walker SE. Milwaukee shoulder with massive bilateral cysts: effective therapy for hydrops of the shoulder. J Rheumatol 1997;24: therapy for

9 Fleish H, Schiller D, Maerki J, Frossard I. Inhibition of aortic calcification by means of pyrophosphate and polyphosphate. Nature 1965:207:1300-1.

$10 \mathrm{Xu} \mathrm{Y,} \mathrm{Cruz} \mathrm{TF,} \mathrm{Pritzker} \mathrm{KPH.} \mathrm{Alkaline} \mathrm{phosphatase}$ dissolves calcium pyrophosphate dihydrate crystals. J 991;18:1606-10.

11 Plumb JA, Milroy R, Kaye SB. Effect of the $\mathrm{pH}$ dependence of 3- (4,5-dimethylthiazol-2-yl)-2,5-diphenyl-tetrazolium bromide-formazan absorption on chemosensitivity determined by a novel tetrazolium-based assay. Cancer Res 1989;49:4435-40.

12 Karnovsky MG. A formaldehyde glutaraldehyde fixative of high osmolarity for use in electron microscopy [abstract]. J Cell Biol 1965;27:137A.

13 Frisch MJ, Trucks GW, Schlegel HB, Gill PMW, Johnson BG, Robb MA, et al. Gaussian 94. Pittsburgh: Gaussian Inc, 1995 .

14 Cini R, Musaev DG, Marzilli LG, Morokuma K. A molecular orbital study of complexes of zinc(II) with imidazole and water molecules. Journal of Molecular Structure and water molecules. Journal of

15 Cini R. A molecular orbital study of complexes of zinc(II) with sulphide, thiomethanolate, thiomethanol, dimethylthioether, thiophenolate, formate, acetate, carbonate, hydrogen carbonate, iminomethane and imidazole: relationships with structural and catalytic zinc in some metallo-enzymes. J Biomol Struct Dyn 1999;16:1225-37.

16 Cini R, Chindamo D, Catenaccio $M$, Lorenzini S, Marcolongo R. Density functional geometry optimization and energy calculations of calcium(II)-triphosphate complexes. Polyphosphates as possible dissolving agents for calcium pyrophosphate dihydrate crystals in chondrocalcinosis disease. J Biomol Struct Dyn 2000;18:155-68.

17 Lusby EW Jr, Laughlin CS. The metabolic properties of acid soluble polyphosphates in Saccharomices cerevisiae. Mol Gen Genet 1980;178:69-76. 